\title{
Aproximación al proceso histórico de ASENTAMIENTO, COLONIZACIÓN Y PRODUCGIÓN EN EL DELTA DEL PARANÁ
}

Guido P. Galafassi*

\section{Introducción}

Intentar reconstruir la historia del Delta del Paraná implica reconstruir un proceso jalonado de acontecimientos dispares, de proyectos intensos y entusiastas seguidos de éxitos como de fracasos. La región del "Carapachay" (como la llamaba Sarmiento) fue objeto siempre de cierta valoración un tanto idílica con respecto a sus cualidades y potencialidades. El propio Sarmiento visualizó a estas tierras (según lo manifiesta su nieto Augusto Belin) como destinadas a ser un rival en producción del valle del Nilo. La alta productividad de las islas y la exuberancia de su vegetación no pasaron desapercibidas por el entonces senador quien manifestó en una sesión de la Cámara su contribución al cultivo de estas tierras por parte de los colonos, quienes "han enterrado en ella millones de pesos (y) las han convertido en una maravilla...El ferrocarril a San Fernando, poniendo en contacto las islas con la ciudad de Buenos Aires, devolverá esos capitales absorbidos y creará un Edén de vegetación a las puertas de Buenos Aires". (1)

Pero este alto entusiasmo se vería contradictoriamente comprobado en el tiempo en una región que, al día de hoy, soporta una fuerte crisis de producción y una alta tasa de emigración de sus pobladores, junto a una descomposición de los sujetos sociales característicos de sus épocas de máximo desarrollo. Cómo explicar estos procesos. La respuesta es compleja y contribuyen a su resolución numerosos

* Becario Post-Doctoral, CEIL-CONICET. 
factores propios de las características de estas tierras y su sociedad, como también fundamentalmente de las relaciones con la región y la sociedad nacional en la cual se insertó diferencialmente a través de los distintos tiempos históricos.

\section{Delta y análisis histórico}

Los pocos estudios referidos a esta zona consisten predominantemente en diagnósticos sincrónicos que no contemplan la trayectoria de la dinámica social, o en trabajos focalizados en temas específicos de realidades parciales.

Estos no pueden explicar el desarrollo seguido por el proceso social que se ha constituido siempre alrededor de ciertas variables fundamentales: ambiente natural conformado por interacciones permanentes entre un medio terrestre y uno acuático; proximidad al centro metropolitano de Buenos Aires; origen predominantemente europeo de la población; carácter marginal de sus tierras en cuanto a su cotización en el mercado, pero de una alta productividad, si bien con fuertes limitaciones ambientales; $y$, una producción directamente vinculada a la explotación de recursos naturales o actividades agropecuarias implantadas en base tanto a las condiciones del mercado como a la oferta ecosistémica.

Es a través del análisis del proceso histórico, rastreando las vidas, trabajos, pensamientos, luchas, formas de subsistencia, estructuras productivas, etc., que puede aproximarse a una comprensión más global de la trama de hechos sociales que dan forma a esta región.

La formación social del presente constituye el resultado de la articulación de estas variables a lo largo del tiempo. Por lo tanto, descifrar el pasado ayuda a comprender la situación presente. Esto último es el objetivo de este trabajo. Es un intento para servir de guía, de acercamiento a la problemática regional, que permita orientar en la búsqueda de explicaciones más precisas y puntuales. No constituye, por supuesto, un estudio histórico acabado, sino sólo una aproximación inicial a la complejidad de situaciones que se articulan en esta original región.

Se han consultado diversas fuentes secundarias de época, desde relatos de viajeros y cronistas hasta ensayos literarios, pasando por informes de diferentes actores de la región. También se han consultado los pocos trabajos científicos, o los un poco más abundantes diagnósticos técnicos (con intenciones de aplicación) existentes. Además, y fundamentalmente para el último período, se ha recogido el testimonio de pobladores actuales o pasados, en base a entrevistas semiestructuradas con aplicación de un cuestionario de preguntas abiertas.

$\mathrm{El}$ recorte regional que merece la atención en este trabajo responde a una necesidad metodológica equivalente a la periodización del análisis histórico (por otra parte también utilizado aquí). Los límites se han establecido en base a fenómenos de cierta homogeneidad interna, como son los dados por el medio natural que definen a la región desde un punto de vista formal. Pero también, desde una óptica 
funcional, podemos encontrar un conjunto que funciona como tal desde el punto de vista económico o social, con cierta independencia de los parámetros físicos. Por supuesto que esta independencia sólo es válida a los efectos conceptuales recién esbozados, porque entender el proceso histórico de una región equivale a explicar el proceso de articulación sociedad-naturaleza (sin caer en explicaciones simplistas del tipo de las del determinismo y posibilismo ambiental). ${ }^{(2)}$ Sino, por el contrario, superando la dicotomía e integrando ambos términos de la relación en un proceso dialéctico de construcción y reconstrucción constante de un medio histórico, es decir "la historia humana comprendida como siendo, a la vez, prolongación y ruptura en relación a la historia natural" (Cardoso y Brignoli, 1987).

Para esto es necesario un marco explicativo que pueda darnos cuenta del funcionamiento de la formación social en donde se insertan los diferentes esquemas productivos. A cada uno de estos esquemas productivos se los puede representar por "actores tipo" que aparecen, se desarrollan, consolidan o destruyen durante el proceso histórico del desarrollo regional, y que responden a un sistema productivo específico, cuyas relaciones con otros sistemas productivos, tanto del mismo como de otros marcos regionales, se van modificando cuantitativa y cualitativamente (Galafassi, 1993).

En principio podríamos definir tres grandes etapas en la historia regional del Delta desde la "conquista", y posteriores al período de ocupación indígena, cuyos primeros habitantes habrían sido: los guaraníes en el Bajo Delta y los chanaes en el Delta Antiguo. ${ }^{(3)}$ La ocupación criolla y europea de estas tierras (siglos XVIII y XIX) marca el comienzo del proceso de construcción de la actual conformación regional. Sin asentamientos permanentes de importancia, estuvo basado en la extracción directa de los recursos naturales. Un segundo período (fines del siglo XIX y principios del $\mathrm{XX}$ ) es donde se inicia el gran proceso de transformación del medio natural, con asentamientos permanentes y el cultivo intensivo de frutales por parte de pequeñas unidades familiares. Y un tercer y último período que comienza a mediados de este siglo y donde lo que varía fundamentalmente es el tipo de producto primario, pasando a la producción casi exclusiva de forestales, con mayor transformación del ecosistema y con un gran proceso de emigración de población, (como consecuencia del cambio productivo) y aparición de unidades productivas de tipo "empresa". En esta etapa se producen cambios en el contexto con el cual el sistema Delta interacciona, lo que repercute en el tipo y forma de las producciones y consecuentemente en la manera en que se utiliza e interviene sobre el medio natural.

\section{Ocupación criolla y europea}

Para esta primera etapa los datos que se han podido recoger tienen un fuerte carácter de dispersión y subjetividad. Cronistas de época y visitantes ocasionales que 
recorrieron la región junto a primeros pobladores han testimoniado sus pareceres en diversas fuentes: notas periodísticas, informes técnicos, obras literarias, relatos de viaje, etc. El fuerte hincapié que se hacía en los datos anecdóticos y llamativos, impide construir una idea acabada de la estructura social del momento. De todas maneras se puede esbozar una aproximación a realidades y procesos parciales que se consolidarán en las etapas siguientes, para las cuales se cuenta con una información cuantitativa y cualitativamente superior.

Un primer interrogante a responder es qué pasó con las escasas poblaciones indígenas de la región. En los documentos que se conservan sobre la conquista y ocupación de estas tierras no se ha podido encontrar grandes datos sobre su relación con los asentamientos humanos primitivos. ${ }^{(4)}$ Por cierto es escasa o casi nula la información que existe en la bibliografia (a diferencia de las otras regiones como Pampeana, Patagonia, NE o NO) sobrc el destino final de los asentamientos indígenas, que podrían dar cuenta hasta dónde cstas culturas tuvieron influencia en la posterior ocupación criolla o europea. Las hipótesis son varias, desde su exterminio o éxodo, hasta su asimilación a la población inmigrante. De cualquier manera, lo que queda bien claro es el carácter netamente diferente de estas poblaciones con los sistemas sociales que ocuparon la región después de la conquista.

Es en esta etapa, a partir de la colonia, cuando la región del Delta comienza a adoptar lentamente la categoría de sector inserto en un esquema mayor de relaciones económicas y sociales, a medida que la ocupación y organización del territorio del Virreinato se iba efectivizando. Pero las colonias del Río de La Plata se mostraban poco atrayentes para la metrópoli española, tanto por su situación geográfica como por las características propias de la conquista. Casi despobladas y carentes de metales preciosos y yacimientos mineros tenían poco que ofrecer a las corrientes económicas y comerciales del Imperio.

En este esquema, el papel que cumplió la región del Delta en los primeros siglos del período fue absolutamente marginal, siendo más bien un territorio extraño e inhóspito que un área productora de cierta importancia como la que se formó unos siglos después. De cualquier forma, se conformaron las bases de procesos posteriores. ${ }^{(5)}$

Las primeras noticias que se tienen del Delta pertenecen a europeos posteriores a Solís, más precisamente al capitán portugués Pedro Lopes de Souza, que arribando alli el $1^{10}$ de diciembre de 1531 y llamándola "Terra das Carandins", anota en su Diario de Navegasao: "es la más hermosa tierra y la más apacible que pueda ser. Yo traía conmigo alemanes e italianos y hombres que habían estado en la India y franceses: todos estaban espantados de la belleza de la tierra, y andábamos todos pasmados, que no nos acordábamos de volver ...No se puede decir ni escribir las cosas de este río y las bondades de él y de la tierra".(6)

El primer intento de colonización occidental del que se tiene noticias estuvo a cargo de los Jesuitas, quienes efectuaron las primeras plantaciones de frutales que 
luego abandonadas se naturalizaron. Con respecto a esto no quedan rastros en la actualidad, pero bien vale el testimonio de Javier Muñiz en un informe que realizara por cuenta del Gobierno en 1818: "Dos leguas más hacia el Miní se ven las reliquias del establecimien to de los jesuitas, que consiste en resto de tapiales y cerca de ellos hay cidra real(?), membrillos, cañas de castilla y varias clases de duraznos" (Mikler, 1991). Otras fuentes dan cuenta del crecimiento espontáneo de especies cultivadas que provendrían de los asentamientos coloniales de más al norte, "...Charles Darwin encontró islas cercanas a la desembocadura del Paraná recubiertas de naranjos y melocotoneros, brotados de las semillas transportadas por el río" (Crosby, 1988:169); el autor se refiere aquí al libro El viaje del Beagle de Darwin, quien en otra de sus obras señala: "muchas plantas se han aclimatado; prueba de ello, el número de durazneros y de naranjos que crecen en las islas de la desembocadura del Paraná, y que provienen de las semillas transportadas allí por las aguas del río" (Darwin, 1972).

Según Liborio Justo (en la introducción a El Carapachay de Sarmiento) algunos "restos de la presencia de los jesuitas al parecer fueron hallados por los primeros investigadores por el arroyo Pay-Carabí...Aunque también, con el nombre de 'Islas de Paicarabí', se conocía primitivamente a las situadas más allá del Paraná de Las Palmas, hasta arriba de Zárate, y se habla de establecimientos de pastoreo que existían en ellas por los años 1761 y 1762 ". Sin embargo, esto nada tiene que ver con la expansión ganadera que comenzaba por esta época en la región del Río de La Plata y que culminará en la industria del saladero. Este incremento de la explotación vacuna se traducirá en un fuerte aumento de las exportaciones que distanciará cada vez más al Río de La Plata de las economías del interior, subsidiarias de la minería potosina. El Delta, que nunca formó parte del área de influencia de las minas del norte, comenzará, sin embargo, a integrar la órbita de las economías rioplatenses. Esta expansión ganadera generará la larga serie de conflictos con los indios en la frontera sur durante el siglo XIX.

Un dato llamativo que se deduce de las anteriores apreciaciones de distintos viajantes y conocedores del Delta, es la ausencia de conflictos con las poblaciones aborígenes y la atracción especial que causa el paisaje al visitante europeo. La temprana presencia de frutales exóticos en forma casi espontánea, marca la ocupación precaria y no planificada de estas tierras por lo viajeros de la época. La calidad de tierras cuasi vírgenes, en donde algunos cultivos se confundían con la exuberante vegetación original dejará de ser tal a partir de los próximos años, en donde transformaciones en la región influirán sobre los territorios del Delta.

Las características de las colonias del Río de La Plata fueron cambiando lentamente gracias a la apertura ocasional del régimen colonial y sobre todo a la organización en gran escala del contrabando. A partir del siglo XVIII Buenos Aires comenzó a vislumbrar algún crecimiento. Los intereses comerciales británicos convirtieron a la ciudad en puerto de acceso de los productos manufacturados extranjeros y puerto de exportación para el cuero y la carne salada. Así Buenos 
Aires se convirtió de a poco en un nuevo polo de crecimiento hacia fines de ese siglo. En tal sentido comenzó a demandar recursos que solventaran el lento incremento de población. (7)

El "monte blanco", ecosistema originario de las islas del Delta, ${ }^{(8)}$ ya se hallaba alterado hacia el momento de la independencia argentina en los primeros años del siguiente siglo. Dos procesos consecuentes estaban ocurriendo: la introducción (espontánea y deliberada) de nuevas especies, y la explotación forestal desordenada, los que fueron creciendo a lo largo del siglo XIX. La explotación del monte natural tenía como destino la ciudad de Buenos Aires, en donde se utilizaba la madera como leña. La extracción de las especies silvestres se realizaba libremente sin estar la actividad regulada ni controlada de forma alguna. Esto llevó a una degradación y desaparición del recurso originario.

La única intervención del Estado se reducía al cobro de derechos de leña, función asumida por el Cabildo de Buenos Aires, durante el Virreinato las tierras fueron consideradas de uso público y comenzaron a ser extraídas maderas y frutales, convirtiéndose así el Delta en el principal proveedor para la metrópoli de esos productos, con Tigre como puerto concentrador.

Las posteriores noticias que se tienen del Delta son ya de la era republicana y proporcionan datos sobre la utilización ya mencionada que se hacía de estas tierras, fundamentalmente en cuanto a la extracción de madera para ser usada como leña. En un texto de 1835, Alcides D'Orbigny proporciona una muy gráfica descripción del procedimien to utilizado: "En estos lugares y algo más arriba en el Paraná, gran número de carboneros acuden todos los años a hacer su provisión de carbón, llegando a ahumar el país a veinte leguas a la redonda. Su modo de fabricación es de lo más vicioso, por lo que el producto resulta muy malo y se pierde mucha cantidad de madera..., y sin que los torpes explotadores se preocupen mayormente por el daño".

Una información un poco anterior sobre el mismo tema pero referido a la escasa población que realizaba esta tarea nos la brindan los hermanos J.P. y G.P. Robertson, comerciantes ingleses que dejaron testimonio de su viaje de regreso del Paraguay en 1816, en sus Cartas de Sud América. Según estos, los pobladores "eran sujetos de apariencia feroz; el chiripá, largo hasta la rodilla dejaba al descubierto sus piernas tostadas y musculosas, y llevaban un poncho sobre los hombros; las caras ennegrecidas por el carbón y las copiosas y negras barbas, patillas y bigotes, acentuaban la fiereza de su aspecto. Los hornos de carbón, al arrojar un resplandor rojizo sobre aquellas salvajes figuras, dábanles apariencia de asesinos. Habían levantado sus cabañas con ramas y cueros, y allí tenían sus hogares. Muchos estaban acompañados por sus mujeres, tan rudas como ellos; $y$ los pequeños casi desnudos o desnudos por completo, veíanse por ahí jugando cerca de los hornos". (9)

Otro empleo singular que se ha hecho de las tierras del Delta hasta el siglo pasado es señalado también por Alcides D'Orbigny, quien menciona el carácter peligroso del territorio por ser sede de corsarios y piratas. Esta particular caracterís- 
tica es tomada por la tradición oral y escrita e incorporada en sus relatos. Lobodon Garra (1955) menciona que: "forajidos de los más famosos del país tuvieron aquí, hasta cerca de los albores de nuestro siglo, un escenario que les perteneció exclusivamente, donde vivieron a su antojo, casi como los únicos pobladores libres de toda influencia extraña, en una vida primitiva y salvaje, sin más ley que sus instintos y sus armas". Otro autor de fines del siglo pasado, el entrerriano Fray Mocho (1966) realiza una descripción detallada del paisaje y del poblador de las islas del río Paraná, de los hechos que ocurren "en el país de lo imprevisto, de lo extraño, en la región que los matreros han hecho suya por la fuerza de su brazo y la dejadez de quienes debieran impedirlo; en la zona de la república perdida donde las leyes del Congreso no imperan, donde la palabra autoridad es un mito, como lo es el presidente de la república o el gobernador de la provincia".(10)

Este primer período, entonces, se caracterizó por la modificación del monte natural a través de la extracción de frutales y madera, leña y carbón y explotación de la fauna. La población era de origen criollo, con una distribución dispersa y en asentamientos precarios y aislados, sin un régimen de tenencia de la tierra ("tierras de pan llevar") y con una escasa o nula intervención del Estado en la ocupación y la explotación de los recursos. Todo esto le daba un carácter de territorio marginal y fundamentalmente de tránsito.

\section{Asentamientos permanentes y fruticultura}

Una segunda etapa comienza a mediados del siglo XIX. Para esta época la nación comienza a vislumbrar un proceso de cambios, que concebidos por la élite ilustrada, pretendían insertar a la Argentina en el concierto de naciones civilizadas "trayendo Europa a América". Se trató de un proyecto de modernización y transformación integral del país que intentaron poner en práctica los grupos dirigentes, elaborado por pensadores de la organización nacional como Alberdi y Sarmiento, este último ferviente impulsor de la ocupación del Delta. Las nuevas condiciones de la economía mundial estructurada sobre la base de las ventajas económicas comparativas suponían una división internacional del trabajo en donde los países europeos sufrieron un fuerte proceso de industrialización que abrió sus mercados a los productos alimenticios de las naciones templadas de ultramar. Estos sistemas económicos se vieron incrementados por la afluencia de importantes capitales extranjeros. Gran Bretaña, banquero mundial, fue, desde luego, el gran proveedor para Argentina. Se calcula, que hacia fines de 1914 aportaba la mitad de las inversiones privadas en el país. ${ }^{(11)}$ El flujo de inversiones de las metrópolis del viejo mundo hacia las naciones periféricas se vio acompañado también por importantes movimientos de población. La inmigración incluida en la nueva Constitución de 1853, donde se manifestaba "asegurar los beneficios de la libertad ...para todos los hombres del mundo que quieran habitar en el suelo argentino" era deseada por los 
gobiernos. Fue oficialmente fomentada y organizada por una ley, a partir de 1876 , durante la presidencia de Nicolás Avellaneda.

Este proceso tiene su correlato en el Delta, en pocas décadas se produce un poblamiento espontáneo pero continuo de las islas con un carácter más estable a través de nuevas actividades productivas. Hacia 1860, el Delta además de leña produce madera para diversos usos urbanos y rurales, y también pieles de nutria y carpincho, naranjas, duraznos y miel. Algunos testimonios de antiguos colonos dan cuenta también del cultivo de trigo y maíz en las partes altas de las islas (albardones), estrategia que define claramente la técnica de cultivo del área, "en realidad toda la vida primitiva del Delta se desarrolló en los albardones. Allí plantaban los durazneros que durante mucho tiempo se suponían de nacimiento espontáneo" (Mikler, 1991).

Esta nueva ocupación estuvo acompañada de impulsos fuertemente simbólicos que ejerció el paisaje "salvaje" de las islas sobre ciertas personalidades de la época. Entre los ya mencionados escritores es fundamental seguir teniendo presente la atracción que ejercía el Delta sobre Sarmiento. La exuberancia de la vegetación, la facilidad de su crecimiento, propagación y alto potencial productivo de sus tierras se cotejan fuertemente en sus escritos. Al respecto manifestaba: “... y los sauces crecen por encanto, y plantíos de tres años dan productos que hallan pronta colocación en el mercado. Vienen lo mismo las parras, los perales, los nísperos y los demás frutales, Crecen las habas como arbustos, el maíz es negro de puro lozano, y las papas y cebollas alcanzan un desarrollo pasmoso" (Sarmiento, 1974). Esta visión un tanto idílica que remite a un supuesto esplendor y que se repite en diferentes fuentes sólo guarda aproximación con el desarrollo económico seguido en la región en este período, y que además es guardado celosamente en la memoria colectiva de la gente del lugar.

Pero el Delta no se constituye en un área productora de bienes para la exportación. Este rol quedó concentrado casi exclusivamente a la pampa húmeda y el litoral, tan cercanos al Delta, pero tan diferentes en geografía y cultura. Las islas, por el contrario, continuaron generando productos primarios para la vecina ciudad de Buenos Aires, en constante crecimiento; si bien se transformaría radicalmente la magnitud y condiciones de esta producción. La colonización por contingentes de inmigrantes europeos y la constitución de un sujeto social definido, la unidad productora familiar, caracterizarán las próximas décadas de su historia.

Para esta etapa comenzamos a encontrar también datos cuantitativos referentes a aspectos demográficos, productivos y de colonización, lo que nos permite reconstruir una historia en base a información más estructurada, y no simplemente a la opinión de cronistas de época.

Con respecto al marco legal para la colonización y el asentamiento poblacional en las islas tenemos un primer dato con el decreto del año 1856, durante el gobierno del Dr. Rafael Obligado, que concedía tierras para su explotación por 
parte de las municipalidades de San Fernando, Las Conchas (Tigre) y sus vecinos (Latinoconsult, 1972).(12)

Sarmiento (1974), sostenía la idea con respecto a la forma tan discutida de otorgar la posesión que "para poner término a tantas divagaciones e incertidumbre, es que la propiedad de las islas sea otorgada a sus actuales poseedores, sin mensura y sin otras condiciones que aquellas en cuya virtud poseían". Así lo sostuvo en una sesión de la Cámara de Diputados de la Nación en 1886, manifestando que el trabajo sobre las islas otorgaba el verdadero valor a esas tierras y justificaba su propiedad.

Esto se concreta en 1888, cuando la provincia de Buenos Aires sanciona la ley 2.072 que se constituye en el primer cuerpo legal orgánico en la materia, tendiendo fundamentalmente a adjudicar en venta las tierras ocupadas y las cedidas por el decreto antes mencionado. Como resultado de esto, se transfirieron al dominio privado una superficie aproximada al $55 \%$ del total del Delta bonaerense en 45 años de vigencia.

En 1934 se sanciona una nueva ley, la 4.207, que hacía fundamental hincapié en la venta de aquellos terrenos fiscales que tuviesen fácil acceso a vías navegables. Esto demuestra la enorme importancia que desempeñaba el canal tanto como vía de acceso, como desagüe para los campos, elemento central de la estrategia productiva en la región. El resultado fue la privatización de alrededor de 40.000 ha. Posteriormente, en 1954, se sanciona la ley 5.782 que adjudica 14.817 ha. hasta que se promulga la 6.263 en 1960, adjudicándose en venta 10.013 ha (Latinoconsult, 1972).

Con estas leyes se fueron blanqueando sucesivamente situaciones de hecho en cuanto a la ocupación de la tierra, dando como resultado un panorama actual con un poco más de 230.000 ha. adjudicadas, quedando alrededor de 45.000 por completar la colonización.

Algunos de sus colonos, aunque más no sea como visitantes temporarios, reflejan en sus escritos algunas características del lugar y su gente. A mediados del siglo pasado, Marcos Sastre (1858) publica una serie de artículos en donde brinda una revisión un poco idílica de la vida en las islas, $\mathrm{y}$ una abierta defensa del paisaje originario: "siglos hace que estas islas preciosas están entregadas al hacha destructora del leñador indolente y son sin tregua esquiladas por la ciega codicia del hombre inculto, sin el coto de la ley y sin el correctivo reparador de la industria".(13)

El paisaje del Delta comienza a transformarse desde un territorio donde hasta entonces sólo existían algunas pocas personas en pequeños huertos, a una sucesión de extensiones desmontadas y plantadas con sauces, álamos, frutales y hortalizas en una magnitud antes no conocida. Al respecto señala Santiago Albarracín en un folleto de la época que "el Delta, compuesto de un archipiélago de islas que ha permanecido algunos siglos desiertas, ha empezado a poblarse vertiginosamente a tal punto que, de un momento a otro, Buenos Aires, ha podido agregar a su mapa 
un departamento nuevo, en el que instantáneamente se han aglomerado capitales por millones y una de las poblaciones más consumidoras del Estado".

La ley de islas de 1888 que da comienzo a las mediciones y ventas, promueve fuertemente la colonización regular del Delta. El nuevo proceso de poblamiento cuenta con un aporte de inmigrantes de gran envergadura y quizá el de mayor diversidad: ucranianos, húngaros, polacos, italianos, españoles, franceses, rusos, vascos, etc. formaron comunidades que en general se agrupaban por países de origen en cursos de agua determinados. Esta ocupación del territorio por inmigrantes no constituyó un hecho aislado, sino que por el contrario se inscribe en los dictámenes que para la época se seguían desde la dirigencia política que se inspiraba en el proyecto "civilizador" de los ideólogos de la organización nacional. La Europa de la revolución industrial que generó una disponibilidad internacional de hombres y capitales, tuvo en el Delta uno de sus puntos de llegada. Si bien en mucha mayor proporción de mano de obra que de capitales. ${ }^{(14)}$

Este proceso de poblamiento continúa hasta la década de 1930 , momento en que el Delta alcanza la mayor población (25.000 habitantes) iniciándose la declinación a partir de la década del 40 por razones fundamentalmente de mercado y climáticas.

Para mediados del siglo pasado se contabilizaban aproximadamente un poco más de 2.000 personas viviendo en forma permanente en las islas del Delta, produciéndose un gran crecimiento hacia fines de ese siglo y principios del XX, llegando a superar los 10.000 habitantes. ${ }^{(15)}$

Según diversas fuentes, este crecimiento demográfico continuó hasta el año 1940, en donde alcanzó una población estimada en 25.000 habitantes, con una densidad de población de $5,9 \mathrm{hab} / \mathrm{km} 2$.

La producción de frutales en unidades productivas de tipo familiar fue lo característico de este período. La organización del tuabajo basado en la participación de la mano de obra de los integrantes de la familia, más el aporte de asalariados permanentes o temporarios, se conjugan con técnicas productivas que utilizan mínimamente maquinarias, tanto por las características del terreno, como por el tipo y tamaño de la explotación.

Estas explotaciones frutícolas se desarrollaron con los primeros colonos europeos asentados en el área. Las mayores concentraciones se localizaron en las secciones $1^{\circ}, 2^{\circ}, 3^{\circ}$ y $4^{\circ}$, correspondientes a los partidos de Tigre, San Fernando y Campana. Conjuntamente a los frutales de verano de hojas caducas (pepita y carozo) como el manzano, ciruelo, durazno, membrillo y peral, se cultivaban especies de invierno (citrus) como el naranjo y limonero, de hojas perennes. Lo común era la práctica de una fruticultura mixta con frutales de verano e invierno. La totalidad del producto se destinaba al mercado interno, y principalmente al Gran Buenos Aires.

Las leyes de ocupación y la instalación de inmigrantes condujeron a un asentamiento estable con producción para el mercado. La cosecha anual de frutales se 
comercializaba a través del puerto de Tigre, llamado justamente "Puerto de Frutos". El Delta funcionó mucho tiempo como el único proveedor de frutas del área metropolitana. La intervención humana sobre el medio se profundizó notablemente a través de la intensificación y diversificación agrícola que dio como resultado el desarrollo de la fruticultura. La modificación del medio natural fue muy notable, reemplazando completamente la cubierta vegetal que cubría las islas, por montes de frutales. La productividad era muy alta, debido a las características ambientales (alta fertilidad por el aporte continuo de materia orgánica que depositan las periódicas crecidas del río). ${ }^{(16)}$ La degradación de los ecosistemas naturales se dio entonces, en términos de desaparición de la comunidad vegetal, pero no en términos de pérdida de rendimiento productivo. Esto se debió seguramente al tipo de unidad productiva y a sus técnicas de intervención. En efecto, estas se basaban en la utilización de la ciclicidad natural del ecosistema con sus pulsos y ritmos de subsidios a través de las inundaciones periódicas. ${ }^{(17)}$ Las modificaciones del terreno perseguían la mayor eficacia en la adaptación al mismo, a través de la utilización de los ritmos naturales en el proceso productivo. Sin impedir las crecidas sobre las islas que aportan el subsidio energético en forma de sedimentos y materia orgánica, se encauzaba para su mejor provecho, "sistematizando" el terreno e incorporando el natural desnivel de las aguas en las estrategias de producción. La existencia, en esta etapa, de pequeñas explotaciones de carácter familiar, con un muy bajo grado de tecnificación, alteró el ecosistema sólo en su estructura florística. El proceso de trabajo con un muy reducido empleo de maquinarias y el tipo de producción basado en la rotación lenta de capital (las plantaciones se renovaban cada varias décadas) determinó una alteración baja en la estructura y dinámica de este ecosistema. El tipo de explotación de poca extensión y atendida principalmente por los miembros familiares, permitía un control más directo de las tareas de intervención. El trabajo se distribuía entre los componentes del grupo doméstico, contratándose trabajadores asalariados para las tareas estacionales, con total supervisión del jefe de la familia (Galafassi, 1994).

En la unidad familiar isleña se verifica la generación de un producto con cierto valor de cambio que se colocará en el mercado, y que se produce a través del trabajo de todos los miembros de la familia, atendiendo también a las necesidades de reproducción de sus integrantes. Es posible definirla como una unidad en donde los miembros en su conjunto trabajan para la producción social de bienes que serán colocados en el mercado. ${ }^{(18)}$ En este trabajo social productivo intervienen todos los integrantes, cumpliendo cada uno un rol predeterminado que está en función del tipo de tareas a realizar y también de cierto arreglo a valores comunes. La división del trabajo por sexo y por edad determina también una distribución específica del mismo que hace ocuparse fundamentalmente a mujeres y niños del trabajo doméstico (y en forma temporaria de la producción agrícola), y a los hombres jóvenes y adultos, de las operaciones concernientes a la producción de bienes para el intercambio. 
En esta producción de bienes y servicios para ser colocados en el mercado, se ponen en operación determinados procesos de trabajo que involucran la transformación de recursos para generar productos con un cierto valor de cambio. Para esto, la familia isleña se constituye con sus miembros en conjunto para participar de dicho proceso, a diferencia de otros casos (familia urbana) en que participan los miembros de la familia en forma individual. (19)

La constitución de esta unidad productiva familiar se dio a principios de siglo a partir de la compra de tierras por parte de inmigrantes llegados a Buenos Aires, proceso que se facilitó debido al bajo precio de las mismas. ${ }^{(20)}$ Es muy común entre los pobladores escuchar cuando el abuelo llegó al Delta y comenzó su explotación en pequeñas quintas en donde trabajaba toda la familia. Otro caso muy difundido es aquel en que se comenzaba como peón de alguna quinta frucícola y a través de un proceso de ahorro se pasaba posteriormente a ser propietario de su propia tierra, otra vez facilitado por el bajo precio de la misma.

El incremento demográfico del Delta se correlaciona con el aumento en la producción frutícola. De esta manera, la década del 40 marca el pico máximo de producción, a partir del cual comienza a decaer. Para el año 1937 se computaron un total de 17.500 ha. cultivadas con frutales (4.3\% manzano, $18 \%$ membrillero, $13 \%$ durazno, $11 \%$ ciruelo, $7 \%$ peral y el resto para limonero, naranjo y mandarino). Para el año 1942 se observa un leve aumento con un total de 18.200 ha. ( $38 \%$ manzano, $18 \%$ membrillero, $11 \%$ naranjo, $10 \%$ ciruelo, y el resto para duraznero, peral, limonero y mandarino. A partir de esta fecha la producción comienza a decaer bruscamente, y en el Censo de 1952 se registra una superficie cultivada de 9.300 ha. - la mitad del censo anterior- ( $43 \%$ manzano, $20 \%$ ciruelo, $12 \%$ peral).

\section{Forestación y emigración}

Este paisaje caracterizó al Delta completamente hasta mediados de este siglo, a partir del cual la competencia de otros mercados (Alto Valle, San Pedro, etc.) inició el proceso de decadencia de la producción frutícola, que encontró en otras zonas mejores ventajas comparativas al insumir menores costos de inversión. El mejoramiento de las comunicaciones privó al Delta de las ventajas que suponía su cercanía a la Capital Federal para la provisión de frutas. ${ }^{(21)} \mathrm{El}$ abandono continuo de la producción y las quintas, la emigración del grupo familiar en su totalidad y el surgimiento de la forestación como única actividad económica, caracterizan el último período que se extiende hasta el momento presente.

Esta etapa que se caracteriza a nivel nacional por el inicio de la industrialización sustitutiva de importaciones llevó al desarrollo de la actividad frutícola en otras áreas del país. La expansión industrial que se lleva a cabo en forma desigual, posibilitó la producción de cultivos industriales y hortícolas en el interior del país. De esta manera se logró la consolidación de ciertas áreas caracterizadas por la 
agricultura de oasis en muy pequeñas explotaciones. Estas áreas de cultivo bajo riego, que entre otros, producían frutales, constituían tanto por las condiciones agrológicas, tecnología aplicada y calidad de los productos, francas competidoras de la fruticultura familiar del Delta. La caída de la producción lleva a una transformación de la estructura demográfica.

La década del 40 marca un giro en la corriente migratoria, esta se invierte, emigrando principalmente población joven, pero se compensa levemente con otro aporte inmigratorio proveniente esta vez del interior del país (Buenos Aires y Entre Ríos), pero disminuye hacia los años 60. En efecto, ya en el Censo de población de 1960 se nota una disminución de pobladores. Los guarismos arrojaron 22.000 habitantes con una densidad de 5,2 hab/km2. El $66 \%$ de esta población se localizaba en las islas bonaerenses y se componía de 8.547 varones (58\%) y 6.165 mujeres (42\%), siendo argentinos $12.014(82 \%)$, y extranjeros, 2.608 (18\%). En el sector entrerriano se radicaba el $34 \%$ restante, compuesta por 4.375 varones $(58 \%)$ y 3.039 mujeres $(42 \%)$.

El CFI estima para el año 1980 un población de 12.200 habitantes localizados en los partidos del Delta bonaerense, lo que lo otorga una densidad de 4,31 $\mathrm{hab} / \mathrm{km} 2$. La tasa anual de variación entre el censo de 1960 y el de 1980 marca un decrecimiento para los dos partidos de mayor población (Tigre y San Fernando) con una tasa de $-1,31$ y -1,61 respectivamente. Campana y Zárate con 2.100 y 1.000 habitantes respectivamente se mantienen casi constantes.

Este proceso resulta paradójico, ya que mientras el crecimiento de Buenos Aires (a la cual el Delta estuvo siempre ligado) da lugar a la conformación del llamado Eje Fluvial Industrial que a lo largo de $400 \mathrm{~km}$. del borde costero litoral concentra casi el $50 \%$ de la población nacional; la porción insular de este espacio geográfico decrece en población y servicios y en la actividad que marcó su desarrollo.

Con respecto al tipo de explotaciones, régimen de tenencia y tamaño de las mismas, derivan del largo proceso de ocupación y utilización de la tierra. La predominancia de unidades productivas de tipo familiar determinó en gran medida un régimen de tenencia y tamaño de la explotación característico, basado en una extensión de pocas ha., atendido fundamentalmente por todos los miembros de la familia.

Según datos del Censo Agropecuario 1988 para los partidos de Tigre y San Fernando (Bajo Delta), el $72 \%$ de las explotaciones posee menos de 50 ha. de extensión, el $15 \%$ son explotaciones de entre 50 y 100 ha., y un $13 \%$ pertenece a explotaciones de más de 100 ha.

Con respecto al régimen de tenencia de la tierra es claramente dominante la propiedad personal o a lo sumo familiar. Las explotaciones en régimen de arrendamiento o aparcería son mínimas sin llegar al 5\% del total (INDEC, 1988). Tiene también escasa importancia la ocupación con permiso o de hecho $(8 \%)$, que se da especialmente en pequeños productores o grupos domésticos aislados con produc- 
ción de subsistencia que suelen ocupar predios fiscales. El resto de la tierra cae bajo el régimen de propiedad personal o familiar $(87 \%)$.

La fruticultura de décadas pasadas que ha dejado paso a la forestación, es complementada en pequeños productores con el cultivo del mimbre, siendo en muchos casos la actividad principal. Al ser de cosecha anual, le permite al productor poder obtener ingresos periódicos mientras espera el turno de corte de la madera. Es un cultivo de bajo grado de tecnificación, pero que necesita un alto aporte de mano de obra, cubierta por el grupo familiar completo. El principal destino de la producción fue originalmente la fabricación de canastos para frutas; al declinar la fruticultura, el mimbre comenzó a ser absorbido paulatinamente por compradores que lo destinan a la elaboración de artículos artesanales para el consumo urbano. Salvo una pequeña cantidad, la fabricación se realiza fuera de las islas, motivo por el cual el producto primario sale de ellas sin un valor agregado. La venta se realiza principalmente en el puerto de Tigre.

Un acontecimiento ambiental, la gran inundación de $1959^{(22)}$ debió acelerar la transformación que llevó al Delta a tener un perfil decididamente forestal, que se inicia hacia los años 50. Por entonces el Estado desarrolla políticas de forestación con maderas blandas en el litoral, que serán destinadas en gran parte al abastecimiento interno de pasta de celulosa.

Entonces, el lugar más destacado dentro de las actividades productivas en el Bajo Delta, lo ocupa en la actualidad la práctica forestal (acompañada en algunos casos por ganadería extensiva) asentada en factores climáticos y edáficos que hacen del área un medio ampliamente favorable para la producción en gran escala de madera de rápido crecimiento, y en especial de maderas blandas. ${ }^{(23)}$ Debido a esto, el ensayo de diferentes especies y variedades es constante, tratando de encontrar las que mejor se adaptan al medio. Las especies ampliamente dominantes son sauces y álamos, existiendo también algunas variedades de pinos, eucaliptos y taxodios.

La importancia creciente de esta actividad surge de la comparación de las cifras de superficies forestadas entre los censos agropecuarios de 1954 y 1969. Para el primer año se da un valor de 79.170 ha. con un $18,82 \%$ del terreno total del Delta (entrerriano más bonaerense), y para el año 1969, esta cifra asciende a 103.320 ha. con un $24,57 \%$ del total del territorio del Delta (Latinoconsult, 1972).(24)

Pero tomando sólo el Delta bonaerense los guarismos son los siguientes, para 1954: 41.600 ha. de superficie forestada con un 15\% del área total; y para 1969: 53.470 ha. con un 19,6\% (Censo Agropecuario 1954, 1969). Posteriormente se detiene este crecimiento, e incluso se registra un retroceso. Según datos del IFONA, en el año 1980 existían algo más de 42.000 ha. forestadas. Pero la gran inundación de 1982-83 produjo pérdidas muy importantes de tal manera que para el Censo Agropecuario 1988 la superficie forestada sólo alcanza las 35.000 ha. (CFI, 1985). De esta superficie el $74 \%$ corresponde a sauces y el $21 \%$ a álamo. A pesar de esta disminución en la superficie plantada, el Delta como monoproductor de salicáceas 
aún constituye la mayor concentración del mundo en cultivos de estas especies en una sola zona ecológica y económica.

Esta orientación hacia los cultivos forestales tropieza, según información proporcionada por los mismos productores, con una estructura fundiaria inadecuada para la práctica forestal, las 10-20 ha. promedio de las quintas frutícolas no permiten desarrollar económicamente el cultivo de salicáceas. Junto con esto, la actividad forestal demanda menos mano de obra que la fruticultura y una atención o cuidado tal que no exige la presencia constante del productor, circunstancia esta que favorece la expulsión de población que se venía dando por la decadencia de la fruticultura. Junto con esto se producen cambios en las relaciones de trabajo dentro y fuera de la unidad productiva en concordancia con las transformaciones de las actividades económicas. La organización del trabajo familiar varía al no requerirse la presencia constante de sus miembros en las explotaciones forestales. Además, al aumentar el tamaño de los predios, se favorece la incorporación de mano de obra asalariada que realiza en forma permanente o temporaria las distintas tareas de la práctica silvícola. Existen también cuadrillas organizadas que recorren las quintas para realizar el corte de la madera, contratándose su trabajo en diversas formas. También se registra un aumento de las maquinarias que se incorporan al proceso de trabajo de estas nuevas explotaciones de tipo empresarial (Galafassi, 1994).

Dado que la "unidad económica forestal" se estima en 150 ha., se favorece un proceso de aumento de tamaño de las explotaciones (Galafassi, 1994). Esto implica la emergencia de grandes productores a costa del abandono y emigración de antiguos fruticultores que venden sus propiedades. Pero en las tres primeras secciones todavía la gran mayoría de los productores son lo que podríamos llamar pequeños y medianos (10-100 ha.), lo que los obliga a adoptar distintas alternativas productivas (mimbre por ejemplo) o sufrir las penurias de una producción insuficiente. ${ }^{(25)}$ En tanto que la situación en parte de la $4^{2}$ sección permitió, en función del mayor tamaño de los predios, la instalación de explotaciones mayores, algunas pertenecientes a grandes empresas que llevan adelante una actividad forestal tecnificada y con el auxilio de endicamien tos para evitar las inundaciones, que permiten, en lo inmediato, obtener resultados económicos más favorables. Esta última situación también se registra en las secciones 1 a 3, aunque en menor proporción. (26)

Pero esta tecnificación y endicamiento implica una transformación profunda de la dinámica natural del ecosistema. Al impedir el ingreso de las aguas al terreno, se impide al mismo tiempo el aporte de subsidios energéticos en materia orgánica al suelo, que es justamente el que le otorga al Delta su carácter de alto rendimiento productivo. Al ser las explotaciones de mayor tamaño, y al emerger las unidades productivas de tipo empresa, se produce una transformación en sus técnicas de trabajo. El objetivo de máxima rentabilidad determina elegir aquellos cultivos más eficientes y utilizar las estrategias productivas más rendidoras. Esto implica transformar y adaptar el medio natural a las exigencias de estos factores, lo que significa un 
notable avance en el grado de transformación del ambiente con respecto a las producciones anteriores. La meta es eliminar las inundaciones creando un medio ambiente más estable que permita el cultivo de especies (álamo, por ejemplo) de mayor productividad económica.

\section{Crisis y transformación}

La decadencia y posterior desaparición de la fruticultura en el Delta trajo aparejado una crisis del sistema productivo en término de cambios en las actividades económicas que repercutieron de manera diferencial sobre un estrato social heterogéneo. A su vez, estos cambios promovieron procesos de diferenciación dentro del estrato social. La emergencia de nuevas actividades productivas produjo la aparición de una tipología de productores diferentes, con técnicas productivas y procesos de trabajo diferentes. Esto implica también nuevas formas de intervención sobre el ecosistema natural, provocando en general modificaciones mucho más significativas.

Esta crisis genera diversas formas de enfrentarla de acuerdo a las características de los distintos tipos de productores.

1) Los productores más grandes, que surgieron a partir de la predominancia de la forestación, son los que están más capacitados para resistir esta crisis. La mayor disponibilidad de tierra, capital y mano de obra, implica una mayor capacidad para producir aquellos productos (álamos, ganado) de más fácil colocación en el mercado y que poseen mayor rentabilidad.

Además, su integración al mercado nacional a través de otras inversiones fuera del Delta, les permite absorber las posibles pérdidas ocasionadas en ciertas temporadas en sus explotaciones en las islas.

La organización del trabajo se estructura primordialmente en base a relaciones de tipo patrón-trabajador.

El mayor aporte de tecnología en sus propiedades, mucho más extensas, permite mejorar la producción cuanti y cualitativamente, generando un mayor proceso de transformación del ecosistema natural para adaptarlo a las exigencias de la producción. Esta práctica termina superando o hasta anulando los condicionamientos que impone el ambiente isleño.

2) Los productores más chicos (que son el remanente de la unidad productiva típica de la fruticultura), que tienen limitada la capacidad de capitalización sufren fuertemente la incidencia de la crisis y los cambios en las actividades económicas. Las respuestas son el abandono de sus quintas, la venta de éstas y la emigración a la ciudad; o en el caso de quedarse, la diversificación de la producción y los ingresos (forestación, mimbre, producción para el autoconsumo, dependencia salarial, ya sea en explotaciones agropecuarias o en empleos públicos, etc.). 
La limitada capacidad de inversión de capital provoca un bajo acceso a la tecnología y un escaso aporte de mano de obra asalariada. Sigue predominando la fuerza de trabajo familiar.

La capacidad de transformación del ecosistema natural es notablemente menor, adecuando sus procesos de trabajo a los condicionamientos ambientales.

3) La unidad productiva familiar, típica de la producción de frutas, está sufriendo fuertes transformaciones al no poder resistir la crisis y el cambio de actividad productiva. Sus alternativas son: crecer y capitalizarse, o subsistir a partir de la diversificación de ingresos. Ambos procesos están generando una descomposición de este sujeto social, tan característico del sistema productivo del Delta durante gran parte de sus historia.

4) La capacidad de intervención sobre el ecosistema natural es notablemente mayor en grandes productores, con un mayor potencial de inversión en términos de tecnologías duras e intensificación del modelo productivo.

Asimismo, la lógica de producción empresarial que se define en base a los incrementos en los rendimientos productivos, determina un tipo de producción (álamo y ganadería) y de técnicas productivas (endicamiento, canalización, etc.) que exige altas modificaciones en la estructura ecológica de las islas y transforma en alto grado la dinámica natural del Bajo Delta, provocando procesos de degradación de diversa magnitud.

En este tipo de explotaciones el ecosistema natural sufre dos tipos de transformaciones, por un lado se elimina toda la cubierta vegetal originaria (esto también ocurre en explotaciones pequeñas) y se reemplaza por forestales y pasturas; y por otro, se anula el régimen periódico de crecidas a través de las obras de endicamiento.

\section{Reflexiones finales}

Esta tierra definida por el ya citado Fray Mocho, como "...la zona de la república perdida donde las leyes del congreso no imperan...", ubicada a las puertas de Buenos Aires y en el corazón de la región agropecuaria por excelencia del país, desarrolló, sin embargo, su historia en términos fundamentalmente diferentes, donde no sólo no "imperan las leyes del Congreso", sino tampoco los factores socioeconómicos y políticos de la pampa y litoral circundantes.

Si consideramos que el aislamiento es un concepto que se determina en base a nociones que exceden el mero parámetro físico, bien vale catalogar a esta región isleña como un área marginal y aislada, definida en base a su situación en el proceso de desarrollo histórico y regional de la nación.

Su nula presencia en el esquema de expansión argentina hacia el mundo de fines del siglo pasado, define claramente su condición de productora de bienes de consumo exclusivos para la metrópoli. Pero es cierto que la época de mayor 
desarrollo del Delta, se debe seguramente a los efectos indirectos del aumento de las exportaciones agropecuarias que motivaron el crecimiento de la ciudad de Buenos Aires. Esta situación de dependencia con la ciudad que perdura hasta hoy (pero que en aquel momento se articulaba a través de una producción de bienes exclusivos), deviene igualmente en una posición de marginalidad tanto por sus características físicas, las cualidades de los asentamientos, y el proceso histórico regional y nacional directamente ligado al mercado económico.

La difícil accesibilidad ambiental de un área de delta implica una tecnología de intervención del terreno y de transporte que nunca estuvieron al alcance de las unidades productoras isleñas.

Esta baja accesibilidad (que incide negativamente en el valor inmobiliario) motivó la instalación en el lugar de pequeños propietarios (generalmente inmigrantes) articulados al mercado regional en condiciones de inferioridad de negociación, acrecentado por un patrón cultural de bajo grado de organización, lo que los llevaba a estrategias productivas y comerciales típicamente individuales.

El ya mencionado proceso histórico de crecimiento económico que tuvo como actores principales a las distintas oligarquías porteñas y provinciales marginó esta región, ajena al interés de estos grupos sociales, y casualmente sin oligarquía propia que la represente dado el recién mencionado proceso de asentamiento. Ligado a esto, la evolución de los medios de transporte colocaba al Delta en condiciones similares cuando la tracción a sangre era el medio dominante, pero el desarrollo del ferrocarril y el posterior crecimiento de la red vial, acercaron a Buenos Aires otras áreas con ventajas comparativas importantes, que no podían manifestarse anteriormente, justamente por la incidencia negativa que implicaba el transporte.

De tal manera, el proceso histórico en la región del Delta está definido en forma importante por parámetros que marcan la marginación y el aislamiento respecto a los procesos socioeconómicos dominantes. La época de auge del desarrollo (fruticultura) se dio, precisamente, porque hubo factores tanto físicos (alta productividad de la tierra) como socioeconómicos (ciudad de Buenos Aires en crecimiento, desarrollo del comercio, accesibilidad relativa, etc.) que permitieron romper temporal y parcialmente estas condiciones de marginación. Concluida esta conjunción de variables, el Delta entra en una crisis que se traduce en un fuerte proceso de emigración y abandono de quintas, disminución importante de la producción, reemplazo de productos y transformaciones de la estructura social que hacen emerger un panorama con diferencias en la tipología de productores mucho mayores a la etapa anterior. La producción de madera en la actualidad, insumo de la industria del papel, mantiene la dependencia con la metrópoli al ser la principal consumidora del producto elaborado, además de articularse a un mercado fuertemente oligopólico, como es el de la industrialización del papel. 


\section{Notas}

(1) Discurso de Sarmiento en una sesión del Senado de 1859 (cfr. "Introducción" por Liborio Justo a: El carapachay de D.F. Sarmiento).

(2) El determinismo geográfico, surgido a fines del siglo pasado y sostenido por F. Ratzel (1882-91) explica las diferencias entre las sociedades humanas a partir de las condiciones diferenciales del medio ambiente natural. Vidal de La Blache (1926) a través del posibilismo, plantea la tesis de relaciones recíprocas entre el hombre y el medio ambiente, cuyo resultado son los "paisajes humanizados". El medio natural ya no determina, sino que posibilita el surgimiento de tal o cual organización social, el ambiente sería una condición permisiva o limitante, una causa necesaria pero no suficiente.

(3) Geomorfológicamente el Delta del Paraná ha sido subdividido en cuatro sectores: 1) Delta Antiguo (ubicado más al norte). Es una zona de mal drenaje, gran cantidad de lagunas, islas de gran extensión con relieve chato, albardones planos, médanos. Las inundaciones que lo afectan producen un efecto de larga duración. 2) Pre Delta (ubicado al noreste). En este alternan albardones y médanos y el escurrimiento superficial es muy bajo. 3) Bajios Ribereños (desde San Pedro hasta San Isidro, al pie de la barranca principal). Están formados por amplias llanuras chatas entre la barranca y el río Luján, en donde el drenaje está impedido. 4) Bajo Delta (ubicado más hacia el este en contacto con el Río de La Plata). Es la región más joven o de reciente formación. El relieve es plano convexo con islas cubetiformes, con albardones periféricos y una cubeta central que recibe gran cantidad de sedimentos.

(4) Un documento importante lo constituye el trabajo de recopilación de Luis María Torres (1911). Se describe a estos grupos como semisedentarios, cazadores, pescadores y recolectores. La propiedad privada no era conocida, y el territorio ocupado era definido y conservado por todo el grupo en defensa de sus fuentes de subsistencia. Algunas citas hacen mención a la enemistad que se originaba entre los guaranies y los otros grupos indigenas, lo que conducía a los primeros a construir sus viviendas en lugares de dificil acceso para los forasteros. Estos son los llamados "cerritos de indios" que ponían a sus moradores al doble resguardo de las aguas y de las asechanzas de sus enemigos (Serrano, 1950).

(5) Contrastando con esta situación, la porción continental del territorio en los vecinos partidos de "Las Conchas" y "De La Costa" (hoy Tigre y San Isidro) tuvieron una importante producción de trigo. En efecto, se calcula que para mediados del siglo XVIII entre el $45 \%$ y el $50 \%$ del cereal se producia en esta zona, siendo la principal abastecedora de Buenos Aires (cfr. Garavaglia, 1993).

(6) Citado por Liborio Justo en la "Introducción" a: EL carapachay (Sarmiento, 1974).

(7) Este crecimiento no significó un cambio radical en el sentido demográfico, pues según cálculos efectuados por especialistas a partir de datos oficiales y de estimaciones de viajeros, la población argentina total sobrepasaba apenas el medio millón de habitantes en tiempos de la Revolución de 1810 (cfr. Mader, 1969).

(8) El "monte blanco" constituia una verdadera selva marginal. Una comunidad muy compleja y rica en especies, que guarda la particularidad de presentar elementos subtropicales que logran avanzar sobre las áreas meridionales del Delta gracias al microclima cálido y húmedo que generan los grandes ríos. Forman bosques de 10-15 metros de altura con especies de hojas anchas y perennifolias y numerosas lianas y epífitas. En la actualidad casi no existe en su formación original, y en aquellos sectores que aún permanece está fuertemente modificada su estructura por gran cantidad de especies invasoras.

(9) Citado en El carapachay, op. cit.

(10) La figura del delito fue asociada en el discurso de la "élite decente" del siglo pasado con la haraganeria, el alcohol y la vagancia. De tal manera que resulta dificil distinguir los límites entre "forajidos" y "manginales" en un contexto signado por valores éticos "higienistas" (cfr. Lvovich, 1993).

(11) Según el estudio británico "Twentieth century impressions of Argentina" citado por A. Rouquié (1981) hacia 1911 el total de inversiones extranjeras en Argentina llegaba a 500 millones de libras esterlinas. En 1913, de casi 1.000 millones de libıs en títulos públicos y privados latinoamericanos cotizados en la Bolsa de Londres, 357 millones pertenecian a la Argentina. Hacia 1900 cerca de la mitad de las inversiones británicas se colocaban en este país. 
(12) En una reconstrucción histórica de San Fernando, del autor A. Gilardoni, encontramos las siguientes referencias: "el 23 de julio de 1856 se facultó al Juez de Paz de San Fernando poder otorgar en propiedad las islas del Delta del Paraná. Es requisito el construir casa y proceder al plantío. En un año se otorgaba la propiedad definitiva. En 1857 igual autorización a los jueces de Paz de Zárate, Baradero, San Pedro y San Nicolás respecto a las islas ubicadas frente a sus respectivas zonas territoriales".

(13) Esta visión idilica también se registra, curiosamente, entre las poblaciones indigenas. Udaondo (1942) citando al célebre etnógrafo Metraux hace mención, en base a referencias históricas y leyendas, a la búsqueda por parte de los guaranies "de la tierra sin mal especie de paraíso terrestre, el Ivy marays. de los apapocuva, donde moraba Nandey, la mujer del creador del mundo. Parece que esta tierra se encontraba al este, al lado del mar".

(14) Nuevamente S. Albarracín cita para la época la presencia de unos 1.500 colonos de origen francés, italiano, inglés, cspañol y criollo, así como de unos 5.000 peones.

(15) En el Primer Censo Nacional de Población del año 1869 se pueden recoger las siguientes cifras de población rural: Las Conchas (Tigre), 1.129; San Fernando, 966.

Para el Segundo Censo Nacional del año 1895, se nota un fuerte incremento de la población rural de ambos partidos, que correspondería fundamentalmente a la sección de islas: 3.996 pobladores para Las Conchas y 5.404 para San Fernando. Incluso aparece un dato interesante: población fluvial existente en los buques anclados en los puertos de la república comprendiendo los que habitan en las islas anegadizas y ouros: Las Conchas 173; San Fernando 389.

Para el Tercer Censo Nacional del año 1914 se nota un leve aumento: Las Conchas, 4.449; San Fernando, 5.900 .

(16) El accionar constante del río Paraná explica en gran parte la estructura y funcionamiento de los ecosistemas deltaicos y sus cualidades productivas. El funcionamiento de cualquier sistema ecológico depende de un flujo continuo de radiación solar que entra al mismo. Pero en el caso del Delta, además de la energía solar, recibe subsidios especiales o pulsos de materia orgánica, sedimentos, nutuientes, agua, semillas y energia hidrodinámica, aportado por el flujo propio del río Paraná y sus afluentes.

(17) Esta contingencia natural está firnemente incorporada al cúmulo de conocimientos del isleño, a tal punto que abundan los testimonios escritos de pobladores con respecto a las inundaciones, describiendo el fenómeno y sus consecuencias, y planteando posibles soluciones (cfr. Mansilla, 1986; Brenner, 1987; Mikler, 1991).

(18) Por trabajo social se entiende aquel que ejecutándose en la unidad de vivienda, su producto es consumido por quienes no pertenecen a ella (cfr. Borsotti, 1978).

(19) Esto no significa que la familia isleña funcione como una unidad en donde prevalezca solamente la concordancia y la solidaridad, sino que también se manifiestan fuentes de conflicto y desarticulación: la emigración de muchos miembros jóvenes abandonando el trabajo en las quintas que se transmitió por varias generaciones, marca una fractura en la continuidad familiar, y es un claro ejemplo de lo anterior. (20) A este respecto, es útil el testimonio dejado por $\mathrm{S}$. Mikler (op. cit.) sobre distintos aspectos del proceso de poblamiento y las prácticas económicas al principio de este siglo, en donde se destaca la importancia de la tierra barata, la colonización por inmigrantes, y el modo de vida de "granjero", con una fruticultura a la "manera antigua" basada en el tuabajo manual.

(21) La producción de frutas bajó notablemente de las 18.000 ha. plantadas en la década de 1940, a unas 2.000 ha. en 1969, quedando en la actualidad un poco más de 200 ha., una cantidad totalmente irrelevante, de la cual gran parte se destina al autoconsumo (Latinoconsult, 1972; INDEC, 1988).

(22) Esta inundación (de carácter extraordinario), se debió al incremento de nivel del río Paraná por lluvias en sus tramos superior y medio, y agravada con una creciente del Río de La Plata por fuerte viento sudeste. La coincidencia de ambas provocó grandes pérdidas en los frutales, como por ejemplo ciertas variedades de limoneros que se perdieron en su totalidad.

(23) La actividad ganadera tiene una importancia secundaria y es de un carácter netamente extensivo. Los establecimientos que combinan forestación con ganado vacuno se los puede localizar en la zona del Río Carabelas $\left(2^{\circ}\right.$ y $4^{\circ}$ sección del Delta bonaerense), y en el Predelta entrerriano se practica fundamentalmente la cría de vacunos en grandes predios. Esta actividad se basa en el aprovechamiento 
de los pastos naturales, con bajo grado de tecnificación, y sometida a los inconvenientes que ocasionan las inundaciones y las dificultades de traslado de la hacienda. En las islas ubicadas en las secciones $5^{\circ}$ y $6^{\circ}$ del Delta bonaerense se encuentran campos de pastoreo que funcionan como complemento de primavera-verano de las explotaciones ubicadas en tierra firme, que llevan la hacienda a pastorear cruzando el riacho San Pedro y el río Baradero.

(24) Complementaria a la forestación, en la producción industrial se destacan las ramas ligadas a la actividad primaria como los establecimientos de tableros de fibra y partículas, pastas de celulosa y papel, y astilleros. Existen también un desarrollo del turismo, y más recientemente los deportes náuticos, concentrados casi exclusivamente en la $1^{\circ}$ sección del Delta bonaerense (cfr. Latinoconsult, 1972).

(25) Se considera pequeños productores a aquellos cuyas explotaciones no superan las $15-20$ ha., con trabajo familiar casi exclusivamente y producción mimbrera y forestal; en cambio los medianos llegan a tener explotaciones de hasta 100 ha. aproximadamente, y suelen contratar mano de obra temporaria para sus producciones exclusivamente forestales, y para la cual existe algún grado de tecnificación con incorporación de maquinarias. Los grandes productores son aquellos que poseen entre 500 y 800 ha., con forestales únicamente y fuerza de trabajo exclusivamente asalariada, permanente y/o temporaria y un alto grado de tecnificación; y luego algunas empresas papeleras que tienen explotaciones de más de 1.000 ha.

(26) La empresa Papel Prensa se instaló en 1978, con Bartolomé Mitre (h) como director. Consume aproximadamente 240.000 toneladas anuales de madera, configurando un mercado cuasi monopólico. Con planta en San Pedro y San Fernando, y plantaciones propias en el río Carabelas, maneja el precio fácilmente. Constituye una de las pocas unidades productivas que posee personal mensualizado en cantidad apreciable,

\section{BIBLIOGRAFÍA}

S. Albarracin, Apuntes sobre las islas delDella Argentino. Cicado en El carapachay (Sarmiento), Eudeba, Buenos Aires, 1974.

C. Borsotti, Notas sobre la familia como unidad socioeconómica, CEPAL, Santiago de Chile, 1978.

R. Brenner, "Nuestro Delta: crecientes y soluciones", en: La Isla, 3 (20), Tigre, 1987.

C.F. Cardozo \& H.P. Brignoli, Historia económica de América Latina, Grítica, Barcelona, 1987.

CFI, Plan de acciones Delta bonaerense. Informe final, vol. 1, Buenos Aires, 1985.

A. Crosby, Imperialismo ecológico. La expansión biológica de Europa, 900-1900, Crítica, Barcelona, 1988.

C. Darwin, Un naturalista en El Plata, CEAL, Buenos Aires, 1978.

A. D'Orbigny, Viaje a la América meridional, t. I., 1835-49.

G. Galafassi, "La relación medio ambiente-sociedad: algunos elementos para la comprensión de su complejidad", en: Revista Paraguaya de Sociologia, año 30, № 86, Asunción, 1993, págs. 127-137.

G. Galafassi, Actividad productiva, onganización laboral y medio ambrienle en el Della del Paraná, Documento de Trabajo, No 37, CEIL-CONICET, Buenos Aires, 1994.

J.C. Garavaglia, "Los labradores de San Isidro (siglos XVIII - XIX)", en: Desamollo Económico, № 128, IDES, Buenos Aires, 1993.

L. Garra, Río Abajo, Anaconda, Buenos Aires, 1955.

A. Gilstfoni, Hitos histónicos de San Fernando, tomo I.

INDEC, Censo Nacional Agropecuario, 1954.

INDEC, Censo Nacional Agropecuario, 1969.

INDEC, Censo Nacional Agropecuario, 1988.

INDEC, Censo Nacional de Población, 1869.

INDEC, Censo Nacional de Población, 1895. 
INDEC, Censo Nacional de Población, 1914.

INDEC, Censo Nacional de Población, 1960.

Latinoconsult, Estudio integral para el desarrollo del Della del Paraná Bonaerense, Ministerio de Economia, Provincia Buenos Aires, 1972.

D. Lvovich, "Pobres, borrachos, enfermos e inmorales: la cuestión del orden en los núcleos urbanos del territorio del Neuquén (1900 - 1930)", en Estudios Sociales, No 5, UNL, Santa Fe, 1993.

J.A. Mader, Evolución demográfica argentina desde 1810 a 1869, Eudeba, Buenos Aires, 1969.

N. Mansilla, "El agua crece sin preguntar", en: La Isla, 2 (16), Tigre, 1987.

S. Mikler, Recopilación de trabajas sobre la geografia, antropologia e historia del Delta del Paraná, Cons. Prod. Delta, Tigre, 1991.

F. Mocho, Un viaje al pais de los matreros, Eudeba, Buenos Aires, 1955.

F. Ratzel, Antropogeographie, J. Engelhorns, Stuttgart, 1881 - 1891.

A. Rouquié, Poder militar y sociedad politica en la Argentina, t. 1, Emecé, Buenos Aires, 1981.

D. Sarmiento, El Carapachay, Eudeba, Buenos Aires, 1974.

M. Sastre, El Temple Argentino, OCESA, Buenos Aires, 1958.

A. Serrano, Los primitivos habitantes de Entre Ríos, Paraná, 1950.

L.M. Torres, Los primitivos hatriantes del Delta del Paraná, UNLP, 1911.

Twentieht sentury impressions of Argentina. Its history, people, comerre, industries and resources, LLoyd's Greater Britain Publishing Company, Londres, 1911.

E. Udaondo, Reseña histórica del partido de Las Conchas, La Plata, 1942.

D.M. Vidal de La Blache, Principles de Human Geography, Holt, New York, 1926. 